\title{
A low-cost partially automated polarimeter for investigating skylight polarisation
}

\author{
C.P. Abayaratne ${ }^{1 *}$, A.V.U.A. Wickramarathna ${ }^{1}$, D.D. Rodrigo ${ }^{2}$ and K.S. Mannathunga ${ }^{1}$ \\ ${ }^{\prime}$ Department of Physics, Faculty of Applied Sciences, University of Sri Jayewardenepura, Gangodawila, Nugegoda. \\ ${ }^{2}$ Central Environmental Authority, 162, Negombo Road, Kurunegala.
}

Revised: 29 June 2015; Accepted: 26 August 2015

\begin{abstract}
Skylight polarisation pattern in general and the positions of the polarisation neutral points in particular are important parameters in atmospheric science. The construction and operation of a low cost partially automated polarimeter for studying the polarisation pattern of skylight in the principal plane of the sun, and some results obtained for the skylight polarisation pattern using the polarimeter are presented in this paper. The polarimeter mainly consists of a collimating tube with a linear polariser and a detector consisting of a light dependent resistor controlled by a microprocessor. The degree of polarisation for a given angular distance from the sun in the principal plane of the sun was measured using the maximum and minimum currents passing through the light dependant resistor (LDR) computed through voltage measurements across the LDR. Positions of the polarisation neutral points and the maximum degree of polarisation were determined graphically using plots of the degree of polarisation as functions of the angular solar distance. The error in the neutral point positions was estimated to be $2^{\circ}$, while that of the degree of polarisation was estimated to be a maximum of $18 \%$. The measurements taken at different times on relatively clear sky days during a period of over one year provided consistent results in fairly good agreement with similar studies carried out using more sophisticated instruments as well as theoretical predictions for a molecular atmosphere. An attempt has also been made to account for any deviations of the quantitative results from those of other studies.
\end{abstract}

Keywords: Atmospheric turbidity, polarimeter, polarisation neutral points, skylight polarisation, skylight scattering.

\section{INTRODUCTION}

Observations on the polarisation of skylight have been carried out as far back as the early years of the nineteenth century. French astronomer Dominique Francois Jean Arago's experiments led to the discovery of the polarisation neutral point in skylight in 1809 , which was later named after him. Arago's neutral point is located a few degrees $\left(20^{\circ}-30^{\circ}\right)$ above the anti-solar point. This discovery opened up the path to a sequence of investigations on the polarisation of skylight. Babinet's and Brewster's neutral points were discovered in 1839 and 1846, respectively following the observations of Jacques Babinet and David Brewster (Konnen, 1985; Hovarth et al., 1998). Babinet's and Brewster's neutral points are respectively located a few degrees $\left(25^{\circ}-30^{\circ}\right)$ above and below the sun for a clear molecular atmosphere. Later in 2001, a fourth neutral point was also discovered beside the anti-sun in the direction directly opposite to the Babinet's neutral point (Hovarth et al., 2002). The fourth neutral point however can only be observed at higher altitudes. Figure 1 shows the positions of these neutral points in the principal plane of the sun, which is the vertical plane passing through the sun and the observer.

Experimental investigation of the scattering and polarisation of skylight was intensified after the theoretical formulation of the Rayleigh theory of scattering of light from atmospheric gases in 1871. Although single scattering does not explain the existence of neutral points, when multiple scattering events are considered, the above neutral points can be theoretically explained. The unpolarised sunlight reaching the earth's atmosphere gets partially polarised after being scattered by atmospheric constituents. The degree and direction of polarisation depend on the scattering angle. The direction of polarisation of the skylight scattered by $90^{\circ}$ is perpendicular to the principal plane according to 


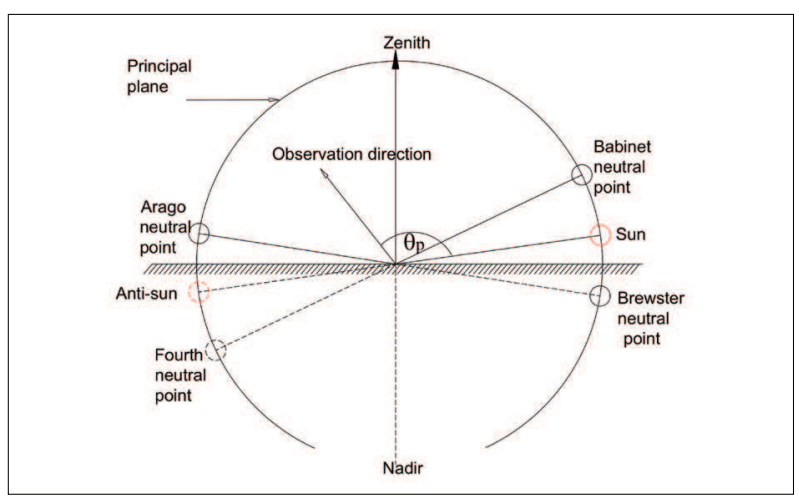

Figure 1: Positions of the neutral points in the principal plane of the sun

Rayleigh's single scattering theory, and is commonly known as positive polarisation. Due to multiple scattering events however, light polarised parallel to the principal plane known as negative polarisation can also be present in the skylight scattered by $90^{\circ}$, reducing the degree of polarisation from $100 \%$ as predicted from single scattering theory. Thus multiple scattering tends to depolarise skylight. Skylight coming from certain directions may consist of equal amounts of positively and negatively polarised light producing unpolarised light or polarisation neutral points. Any changes in the atmospheric composition such as aerosols that increase multiple scattering cause the neutral points to deviate further away from the sun or the anti-sun (Gambling \& Billard, 1967; Hovarth et al., 2002). Much work has been done in order to locate these neutral points experimentally, employing various methods that became increasingly sophisticated with time. Originally, visual polarimeters have been used to locate the neutral points for various positions of the sun carried out throughout the day, which were later developed to detect the neutral points with electronic polarimeters (Hovarth et al., 1998). The most recent investigations of the neutral points have been carried out by full-sky imaging video polarimetry using high-resolution digital cameras, which are employed to take pictures of the sky at a desired time, and digital image processing techniques are then applied to determine the positions of the neutral points (Liu \& Voss, 1997; Hovarth et al., 1998; Gal et al., 2001).

Extensive studies have been carried out by many researchers on the dependence of turbidity on skylight polarisation. Investigations have revealed that the angle between the neutral points and the sun depends on factors such as the position of the sun and the atmospheric turbidity among other factors (Sekera,1957; Holzworth \& Rao, 1965; Gambling \& Billard, 1967; Coulson, 1980; Hovarth et al., 1998). Therefore the locations of the polarisation neutral points provide a sensitive tool to measure the atmospheric turbidity, which in turn could be used as a measure of the extent of air pollution.
In addition to experimental studies, theoretical models have also been developed by many researchers to describe skylight polarisation patterns (Chandrasekhar, 1954; Smith, 2007). Some studies have attempted to relate atmospheric particle size with the neutral point positions (Liu \& Voss, 1997). However, to our knowledge, skylight scattering and polarisation studies have not been carried out in Sri Lanka and their applications are not used in the country to investigate environmental turbidity, probably due to the high cost associated with such studies.

In this paper the construction of a low cost partially automated polarimeter for investigating skylight polarisation, and the results obtained for the variation of the degree of polarisation in the principal plane of the sun with the angular solar distance measured using the polarimeter have been reported. The construction cost of the polarimeter did not exceed 7000 Sri Lankan Rupees (about US\$ 50) and was very low compared to the extremely expensive instruments estimated to cost over US\$ 10,000 used by researchers in developed countries, which made this investigation on skylight polarisation possible. This polarimeter could be employed as an educational tool for university undergraduate students to investigate the polarisation pattern of skylight and its variations with atmospheric conditions. This preliminary study on the polarisation of skylight was conducted in a relatively clear site at the University of Sri Jayewardenepura playground (latitude $6^{\circ} 51^{\prime}$, longitude $79^{\circ} 53^{\prime}$ and altitude $30 \mathrm{~m}$ ). The nearest buildings and trees were located about $50 \mathrm{~m}$ from the measurement site. The experiment was repeated on many fairly clear days during the period from October 2012 to January 2014. Essential steps in the construction of the low-cost polarimeter and its operation are described in the next section and some results obtained using the polarimeter are presented in the results section. An attempt has been made to qualitatively explain the observed deviations in the results from those for a high altitude molecular (Rayleigh) atmosphere in the absence of obstacles.

\section{METHODS AND MATERIALS}

\section{Construction and operation of the polarimeter}

The main part of the polarimeter consists of a collimating tube, a linear polariser and a light dependent resistor (LDR). The collimating tube has a length of $17.5 \mathrm{~cm}$ and the top end of the tube is closed with an end-cap having an aperture of diameter $9 \mathrm{~mm}$. This restricts the light received from a given direction to a cone of vertex angle $3^{\circ}$ giving an uncertainty of $\pm 1.5^{\circ}$ in the angle measurement $\theta_{p}$ between the sun and the observation direction although it is measured with 
an accuracy of one degree using a protractor attached to a manually operated sun-tracker and a pointer attached to the collimating tube. Figures 2 and 3 show a photograph and a schematic diagram of the polarimeter, respectively. The measured angle $\theta_{p}$ is illustrated in Figures 1 and 3.

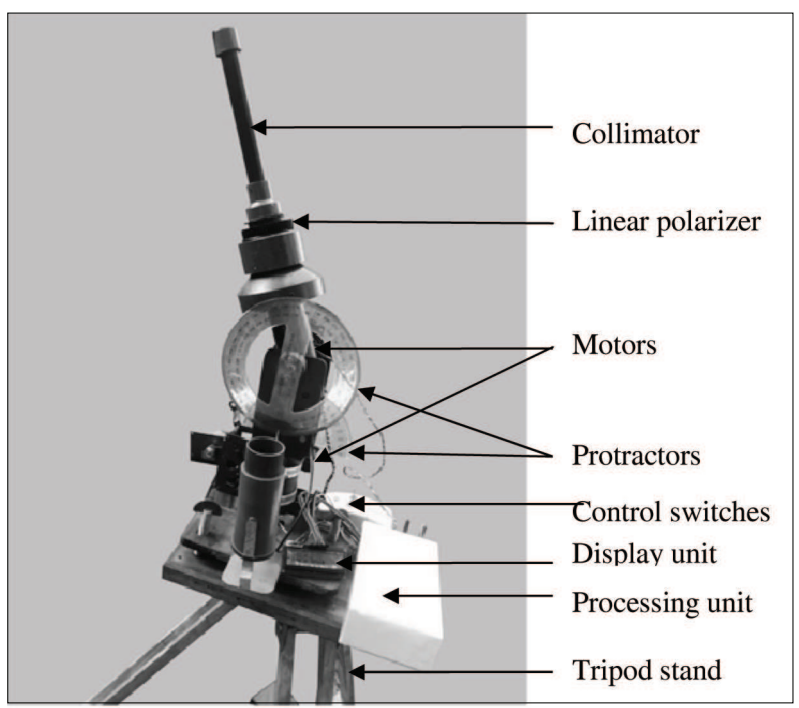

Figure 2: Photograph of the low-cost partially automated polarimeter

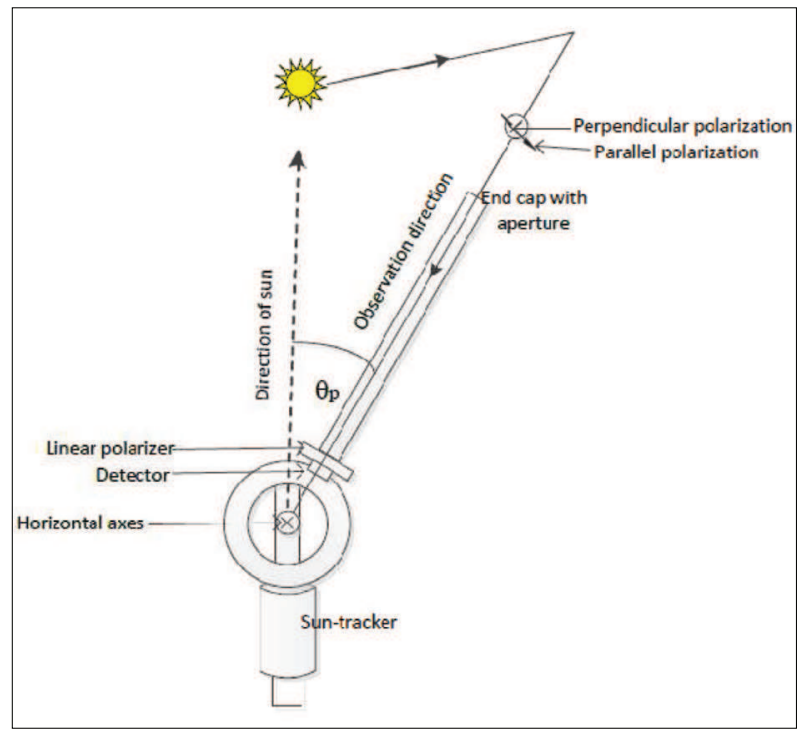

Figure 3: Schematic diagram of the polarimeter

In the operation of the partially automated polarimeter the first step is finding the principal plane of the sun. This was done manually, with the aid of a platform that can be rotated around two axes, one horizontal and one vertical. First, the central axis of the collimating tube and the principal axis of a short focal length convex lens were aligned parallel to one another and the whole unit was turned towards the sun. The image of the sun was first focused on a plane parallel to the lens. The system was then rotated around the horizontal and vertical axes in turn until the center of the image coincided with a point passing through the principal axis of the lens. Once this is done, the collimating tube rotates in the principal plane of the sun relative to the sun-tracker when it is rotated around the horizontal axis. After finding the principal plane of the sun and manually setting the direction between the sun and the observation direction, the rest of the data taking process involving the rotation of the linear polariser and finding the maximum and minimum voltages across the LDR and displaying the output on a liquid crystal display (LCD) was carried out automatically, under the control of the microcontroller based system. The data recording however was done using a voice recorder for further analysis.

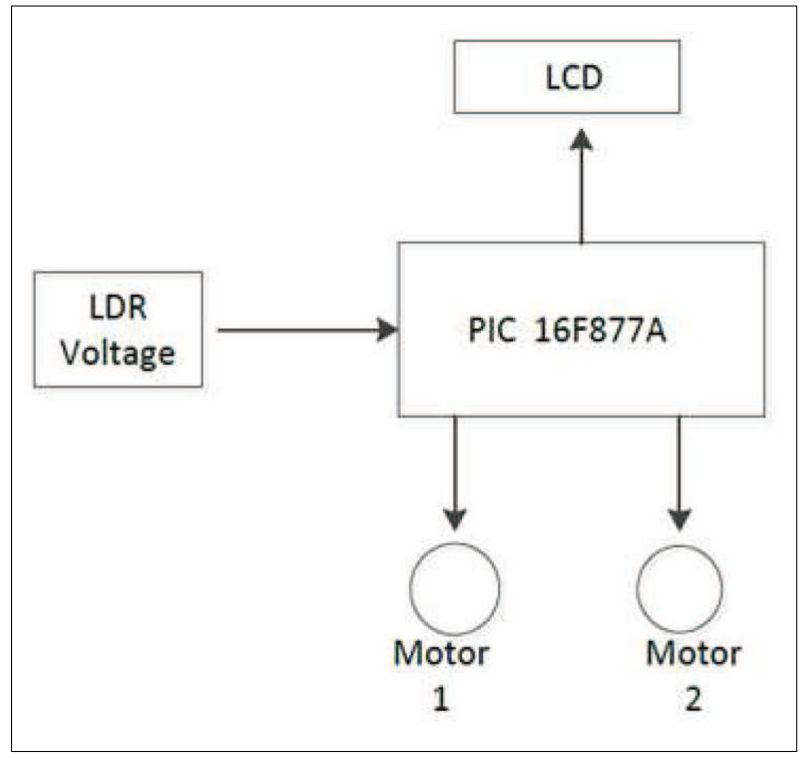

Figure 4: Block diagram of the processing unit

Figure 4 shows a block diagram of the microcontroller circuit. A PIC 16F877A microcontroller was used to control a stepper motor and a gear motor, to collect and process data and to display the results on an LCD. The stepper motor was used to rotate the polariser around the axis of the collimating tube and the gear motor was used to rotate the system consisting of the collimating tube, polariser and the LDR in the principal plane of the sun. (Another gear motor not controlled by the microcontroller circuit was also used to locate the principal plane). A $12 \mathrm{~V}$ battery was used to supply power to the motors and a $5 \mathrm{~V}$ to the LDR through regulators. 
The voltage across the LDR was read out by one of the built-in analog to digital converter (ADC) channels of the microcontroller. Figure 5 shows how the LDR was connected to the ADC. The buffer circuit was used to provide a high impedance between the voltage divider and the ADC input. With a 10-bit resolution and $5 \mathrm{~V}$ range, the ADC had a voltage resolution of $4.88 \mathrm{mV}$. Once the voltage $\mathrm{V}$ across the $\mathrm{LDR}$ is measured, the voltage across the $15 \mathrm{k} \Omega$ resistor is known and therefore the current $i$ (in $\mathrm{mA}$ ) through the LDR, which is proportional to the incident light intensity was calculated from the equation $i=\frac{5-V}{15}$.

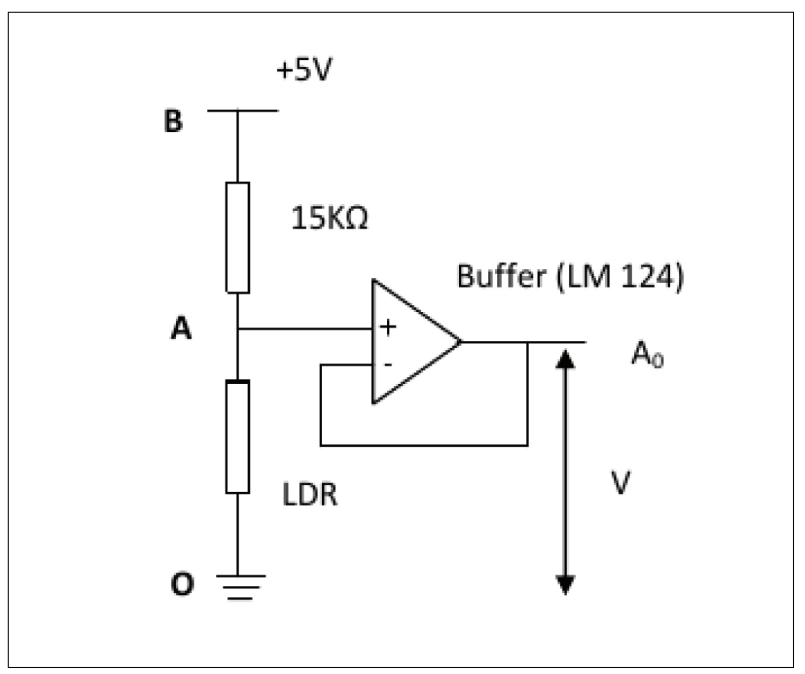

Figure 5: Detector circuit illustrating how the LDR was connected to the ADC

During automated data taking, the microcontroller was programmed to rotate the polariser by $360^{\circ}$ in 52 steps and record the LDR voltage at each step. Upon reaching each step, the LDR voltage was recorded after a 50 ms delay to allow for the LDR response time. At the end of each cycle of the polariser rotation, the maximum and minimum currents through the LDR were determined. This procedure was repeated by varying the angular solar distance in steps of $2^{\circ}$ for all possible angles to cover the principal plane above the sun. The voltage maximum and minimum values measured above were later used to compute the corresponding minimum and maximum currents through the LDR. The degree of polarisation $d_{l}$ was computed from the values of the currents using the formula,

$$
d_{l}=\frac{I_{\max }-I_{\min }}{I_{\max }+I_{\min }}
$$

In the analysis, graphs of the maximum and minimum currents as well as the degree of polarisation were plotted as functions of the angular solar distance in the principal plane of the sun. Positions of the degree of polarisation maxima and minima were read from the graphs. Minima of the degree of polarisation curves indicate the polarisation neutral points.

\section{Linearity of the detector}

The linearity of the LDR detector was checked in the laboratory by letting a nearly parallel beam of light from a $100 \mathrm{~W}$ tungsten filament lamp to pass through two polarisers onto a similar LDR detector connected in a similar circuit. The voltage across the LDR was measured using the ADC of the microcontroller by varying the angle $\theta$ between the two optical axes of the polariser from $0^{\circ}-90^{\circ}$ at $10^{\circ}$ intervals controlling the input intensity. The current was computed as described before. A graph of the current plotted versus $\cos ^{2} \theta$ gave a curve indicating a nonlinear response. The data was later fitted into a straight line through a least square regression fit, which gave a $\mathrm{R}$ squared value of $82 \%$. Therefore the LDR response was assumed to be linear with an estimated error of $18 \%$.

\section{RESULTS}

Some of the results obtained at different times during the period from October 2012 to January 2014, with the use of the partially automated polarimeter described above are presented in this section. Figures 6(a), 6(c), 6(e), 6(g) and 6(i) show the relative intensity variations of the incident light polarised in two perpendicular directions, with angular solar distance indicated by the corresponding maximum and minimum currents on two selected relatively clear days with a very few visible clouds. The upper and lower curves in each of these figures plotted using the maxima and minima of the currents computed using the voltage measurements as described in the Methods and Materials section, are related to the intensity of scattered skylight polarised in a direction parallel and perpendicular to the optical axis of the linear polariser and reaching the detector after being scattered from atmospheric constituents. The currents plotted in the graphs are indicated as a fraction of the highest current observed in the direction of the sun for convenience. Figures 6(b), 6(d), 6(f), 6(h) and 6(j) show the corresponding variations of the degree of polarisation with the angular solar distance. Figures 6 (a) - (f) are based on measurements taken in the morning, while Figures $6(\mathrm{~g})$ - (j) are based on measurements taken in the evening. 


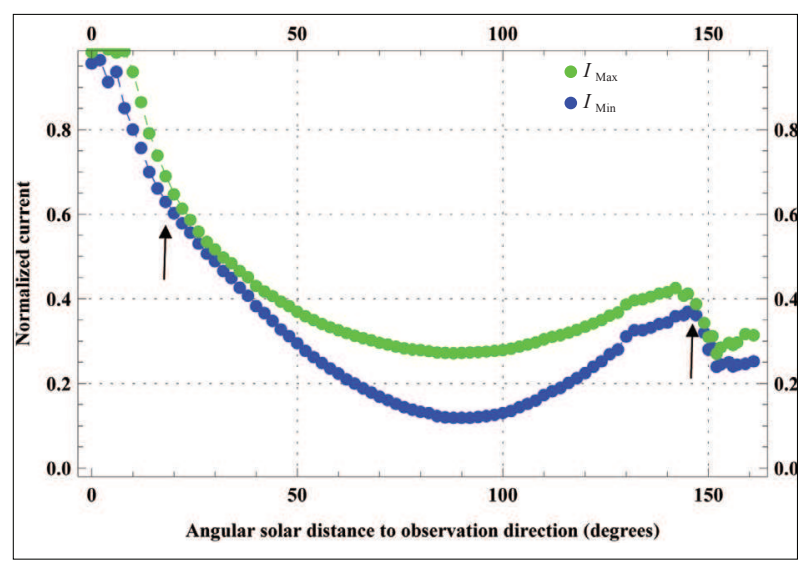

Figure 6(a): Maximum and minimum currents $\mathrm{I}_{\max }$ and $\mathrm{I}_{\min }$ (normalised currents) corresponding to two perpendicular polarisations as a fraction of the respective currents near the sun at various angular distances from the sun measured on 16.01.2013 from $7.24-7.42 \mathrm{~h}$

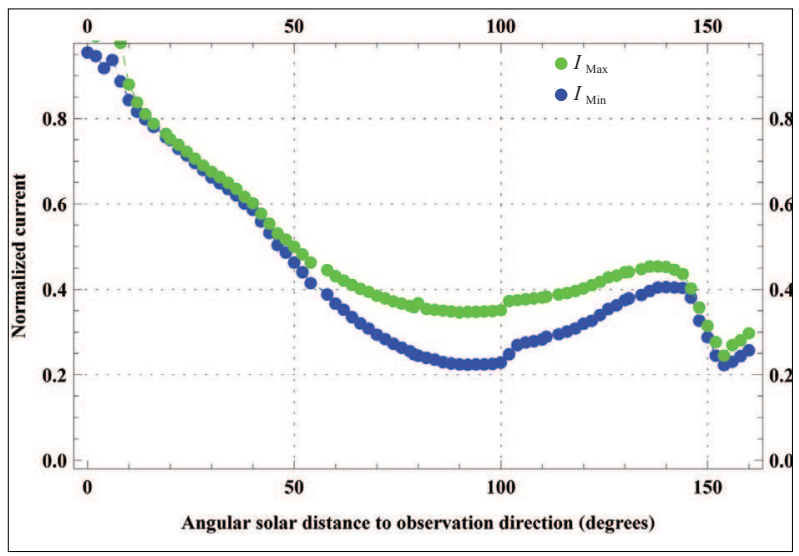

Figure 6(c): Maximum and minimum currents $\mathrm{I}_{\max }$ and $\mathrm{I}_{\min }$ (normalised currents) corresponding to two perpendicular polarisations as a fraction of the respective currents near the sun at various angular distances from the sun measured on 18.01.2013 from $07.44-07.59 \mathrm{~h}$

Each curve of the degree of polarisation illustrates the increase in the degree of polarisation with increasing angular distance from the sun up to a maximum in the degree of polarisation occurring at a solar angular distance close to $90^{\circ}$ as expected. The curves also illustrate the Babinet neutral point shown by the minimum near the sun, and the Arago neutral point shown by the somewhat sharper minimum further away from the sun (close to the anti-sun). These neutral points can also be seen in the maximum and minimum intensity curves where the gap between the maximum and minimum currents is narrowest. The positions of the neutral points

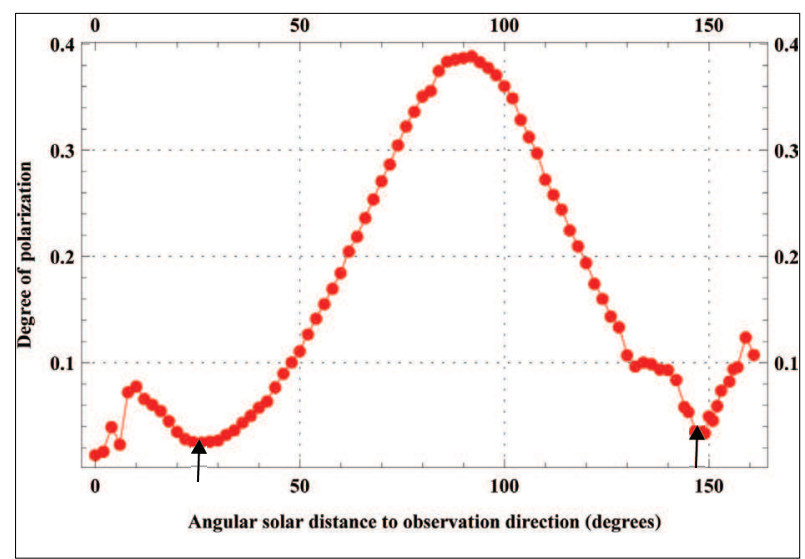

Figure 6(b): Degree of polarisation at various angular distances from the sun measured on 16.01.2013 from $7.24-7.42 \mathrm{~h}$

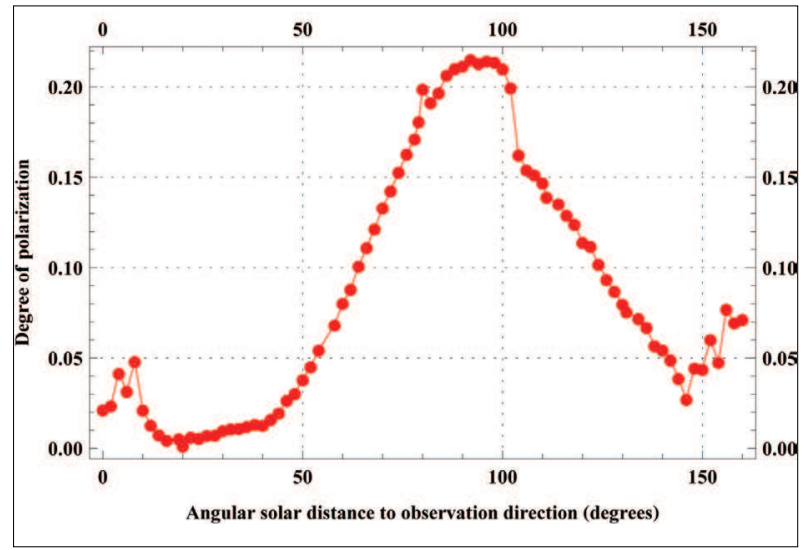

Figure 6(d): Degree of polarisation at various angular distances from the sun measured on 18.01 .2013 from $07.44-07.59 \mathrm{~h}$

are illustrated in graphs 6(a) and (b) by arrows. The maximum degree of polarisation and their locations, and the positions of the Arago and Babinet neutral points read from the graphs are presented in Table 1. The positions of Arago and Babinet neutral points computed theoretically by Holzworth and Rao (1965) and presented in the form of graphs (marked with * in parenthesis) and Arago point positions measured by Liu and Voss (1997) and plotted in their Figure 10 for the spectral frequency $560 \mathrm{~nm}$, were interpolated and used to find the values corresponding to the elevation angles of the sun presented in this paper for purposes of comparison although the conditions in 
Table 1: Measured values of the maximum degree of polarisation, their positions and the positions of the Arago and Babinet neutral points from the sun measured on three selected relatively clear days. On 08.01.2014 a few small thin clouds were observed in the sky.

\begin{tabular}{|c|c|c|c|c|c|c|}
\hline Date & Time & $\begin{array}{c}\text { Elevation } \\
\text { angle of } \\
\text { the sun }\end{array}$ & $\begin{array}{c}\text { Maximum } \\
\text { degree of } \\
\text { polarisation }\end{array}$ & $\begin{array}{l}\text { Observed angular } \\
\text { solar distance of } \\
\text { maximum degree } \\
\text { of polarisation }\end{array}$ & $\begin{array}{c}\text { Observed angular } \\
\text { anti solar } \\
\text { distance to } \\
\text { Arago point }\end{array}$ & $\begin{array}{c}\text { Observed angular } \\
\text { solar distance to } \\
\text { Babinet point } \\
\text { (approximate) }\end{array}$ \\
\hline \multirow[t]{2}{*}{16.01 .2013} & $07.24-07.42 \mathrm{~h}$ & $18-21^{\circ}$ & $35 \%$ & $90^{\circ}$ & $33 \pm 2^{\circ}$ & $25 \pm 2^{\circ}$ \\
\hline & & & & & $\left(36^{\circ}\right)^{* *}$ & $\left(18^{\circ}\right)^{*}$ \\
\hline \multirow[t]{2}{*}{18.01 .2013} & $07.44-07.59 \mathrm{~h}$ & $20-22^{\circ}$ & $22 \%$ & $92^{\circ}$ & $34 \pm 2^{\circ}$ & $19 \pm 2^{\circ}$ \\
\hline & & & & & $\left(37^{\circ}\right)^{* *}$ & $\left(17^{\circ}\right)^{*}$ \\
\hline \multirow[t]{2}{*}{18.01 .2013} & $08.09-08.23 \mathrm{~h}$ & $26-29^{\circ}$ & $21 \%$ & $88^{\circ}$ & $42 \pm 2^{\circ}$ & $20 \pm 2^{\circ}$ \\
\hline & & & & & $\left(40^{\circ}\right)^{* *}$ & $\left(16^{\circ}\right)^{*}$ \\
\hline \multirow[t]{2}{*}{08.01 .2014} & $16.52-17.03 \mathrm{~h}$ & $160-163^{\circ}$ & $21 \%$ & $96^{\circ}$ & $30 \pm 2^{\circ}$ & $26 \pm 2^{\circ}$ \\
\hline & & & & & $\left(36^{\circ}\right)^{* *}$ & $\left(17^{\circ}\right)^{*}$ \\
\hline \multirow[t]{2}{*}{08.01 .2014} & $17.09-17.30 \mathrm{~h}$ & $164-167^{\circ}$ & $15 \%$ & $98^{\circ}$ & $31 \pm 2^{\circ}$ & $25 \pm 2^{\circ}$ \\
\hline & & & & & $\left(19^{\circ}\right)^{*}\left(35^{\circ}\right)^{* *}$ & $\left(18^{\circ}\right)^{*}$ \\
\hline
\end{tabular}

Values in parenthesis for the Arago and Babinet points were obtained by reading to the nearest degree from graphs of Figure 3 of Hozworth and Rao (1965) (marked with *) computed and plotted for a molecular atmosphere for a wavelength of $512.5 \mathrm{~nm}$ and for the Arago point by interpolating the measured values from Figure 10 of Liu and Voss (1977) for a wavelength of $560 \mathrm{~nm}$ (marked with **) measured in Miami, Florida in 1996 for the corresponding sun's elevations.

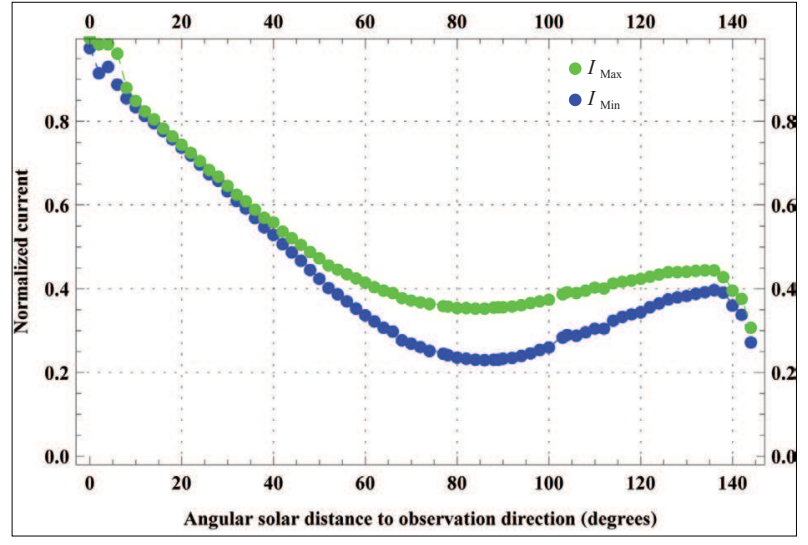

Figure 6(e): Maximum and minimum currents $I_{\max }$ and $I_{\min }$ (normalised currents) corresponding to two perpendicular polarisations as a fraction of the respective currents near the sun at various angular distances from the sun measured on 18.01.2013 from $08.09-08.23 \mathrm{~h}$

the results of our work are not the same. The values given in parenthesis marked with ** from the graphs of Holzworth and Rao (1965) correspond to a spectral frequency of $512.5 \mathrm{~nm}$ for an optical depth $\tau=0.123$ in the absence of ground reflections, while those of Liu and Voss (1977) are for $\tau=0.24$. As can be seen from

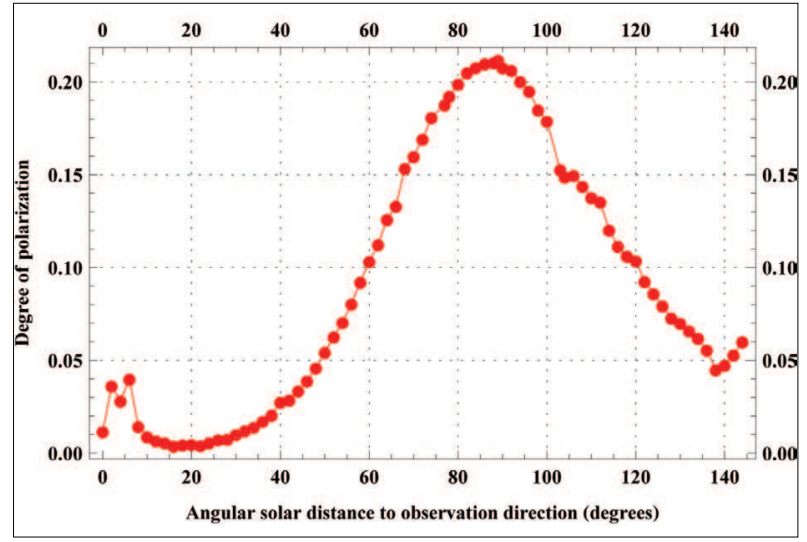

Figure 6(f): Degree of polarisation at various angular distances from the sun measured on 18.01.2013 from $08.09-08.23 \mathrm{~h}$

Table 1, Arago point positions found from our analysis agree fairly well with those of Liu and Voss (1977). The differences are obviously due to the different optical thicknesses, background conditions and aerosol content in the locations where the experiments were carried out. A direct comparison however was not possible as the 


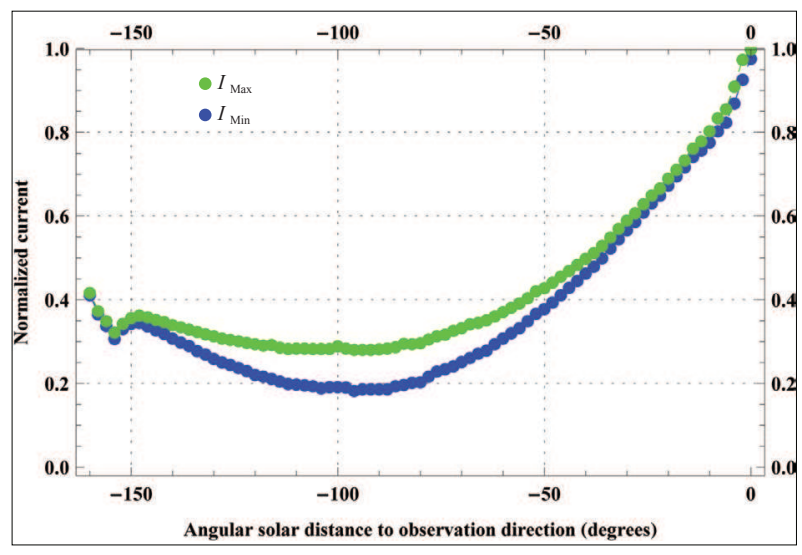

Figure 6(g): $\quad$ Maximum and minimum currents $\mathrm{I}_{\max }$ and $\mathrm{I}_{\min }$ (normalised current) corresponding to two perpendicular polarisations of scattered skylight as a fraction of the respective currents near the sun at various angular distances from the sun measured on 08.01 .2014 from $16.52-17.03 \mathrm{~h}$

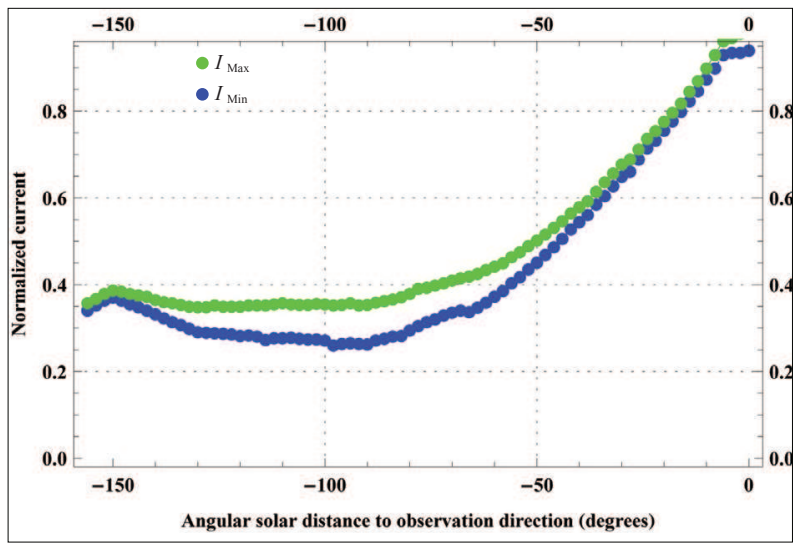

Figure 6(i): $\quad$ Maximum and minimum currents $\mathrm{I}_{\max }$ and $\mathrm{I}_{\min }$ (normalised currents) corresponding to two perpendicular polarisations as a fraction of the respective currents near the sun at various angular distances from the sun measured on 08.01.2014 from $17.09-17.30 \mathrm{~h}$

environmental conditions of the available theoretical or experimental data were different from ours.

A sudden drop in the maximum and minimum currents are observed in Figures 6(a), 6(c), 6(e), 6(g) and 6(i) at large angles. As an example, in Figure 6(a), this drop begins at $\theta_{p}=142^{\circ}$ while the elevation of the sun is about $20^{\circ}$. This corresponds to an observation direction that makes an angle of $18^{\circ}$ with the horizontal plane. Buildings and trees although located at least $50 \mathrm{~m}$ from the measurement site could obstruct some part of the skylight reaching the collimating tube causing

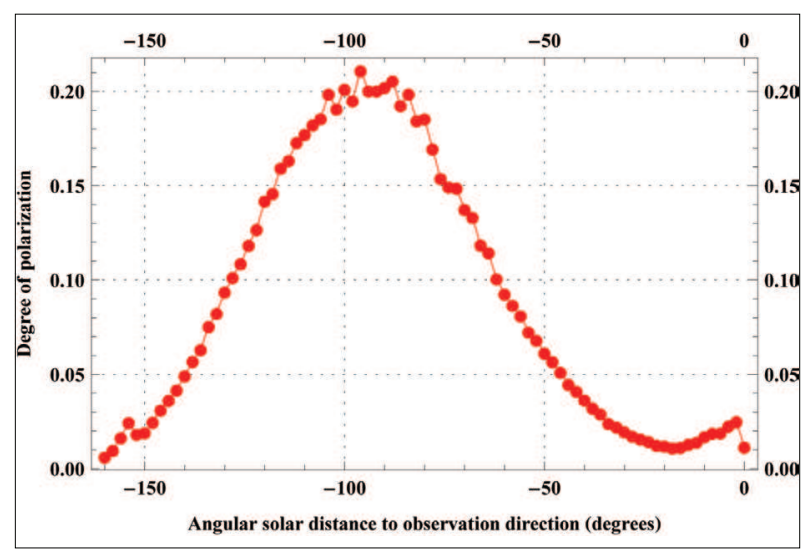

Figure 6(h): Degree of polarisation at various angular distances from the sun measured on 08.01.2014 from $16.52-17.03 \mathrm{~h}$

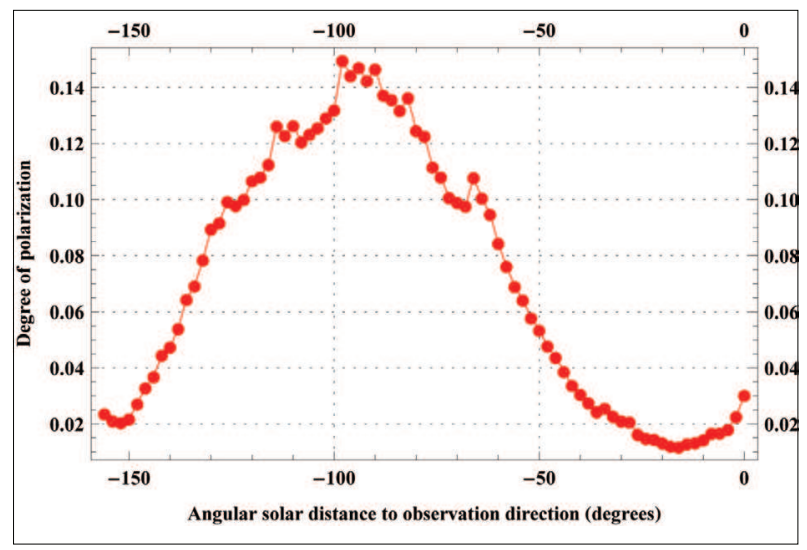

Figure 6(j): Degree of polarisation at various angular distances from the sun measured on 08.01.2014 from 17.09-17.30 h

a reduction in the light incident on the detector. However some scattered skylight reaches the detector along with some light scattered by the buildings, trees and the ground. Due to this mixture of light, the neutral points read from the graphs could be slightly affected. On 08.01.2014 a few small thin clouds were observed in the sky, which could be the reason for the variations in the degree of polarisation in the directions making $70^{\circ}-130^{\circ}$ with the sun. Further investigations in an obstruction free site need to be carried out in order to ascertain the positions of the neutral points more accurately. 


\section{DISCUSSION}

In order to take each set of measurements shown in Table 1, about 16 minutes had to be spent on the average. This could introduce an error of about $1.5^{\circ}-2^{\circ}$ in the measured angles as the sun's position changes by about $3^{\circ}-4^{\circ}$ during this time interval. This error could be easily reduced to half of this by taking measurements only for a rotation of $180^{\circ}$ of the polariser. Further reduction in the error is possible by fully automating the operation of the polariser and reducing the time spent on taking measurements. Errors that could arise in the voltage measurements through any nonlinearity in the response of the LDR to light intensity are assumed to be minimal, in reading the positions of the neutral points from the minima of the continuous graphs. However the effect of the nonlinear response on the maximum degree of polarisation in the table could be significant with an estimated maximum error of $18 \%$.

Positions of the Arago point for a molecular atmosphere under clear sky conditions range between $20^{\circ}-30^{\circ}$ with time of the day, as reported in the literature while those of the Babinet point vary from $25^{\circ}-30^{\circ}$ (Hovarth et al., 1998). The differences in the observed values could be attributed to the atmospheric conditions prevailing at the time and the site where the measurements were taken such as optical thickness, ground reflections (albedo) and atmospheric turbidity, which are quite different from clear sky conditions. The measurement site was about $30 \mathrm{~m}$ above sea level where the optical depth is rather high. Sometimes an occasionally passing cloud interfered with the data in spite of selecting as clear days as possible at the location where the experiments were carried out. The values reported in literature are from higher altitudes with lower optical densities. Therefore the maximum degree of polarisation is rather low, not exceeding $40 \%$. Other factors such as surface reflections from the ground and light scattered from close by bodies as well as aerosols in the atmosphere could also contribute in depolarising effects to decrease the degree of polarisation. The use of white light rather than a narrow spectral band could broaden the neutral point positions. Further studies are planned for a more accurate determination of the positions of the neutral points, and for investigating the effects of atmospheric turbidity and the positions of the polarisation neutral points, specifically those of the Arago point.

The differences in the overall shapes of the graphs plotted using the data from January 2013 and January 2014 could be attributed to the change in the position of the sun as well as that of the principal plane of the sun, both of which vary as the position of the sun changes with time of the day as well as the time of the year and the resulting variations in the background effects. Data of 2013 were taken during the morning hours, while those of 2014 were taken during the evening hours. In the morning hours, direct sunlight is incident on the walls of the white-washed hostel building situated about $60 \mathrm{~m}$ from the measurement site, which gives rise to a strong field of diffused light causing a strong depolarising effect that reduces the degree of polarisation in certain directions than that expected from theory for an obstacle free area. In the evening, the sun is not directly visible from the measurement site as it is covered by the hostel building and the depolarising effect due to the light scattered from the hostel building walls was absent.

\section{CONCLUSION}

The results obtained using the partially automated low cost polarimeter verified some fundamental properties of skylight scattering and polarisation. This makes the partially automated polarimeter a useful educational tool for a developing country to investigate environmental turbidity. The polarimeter could be further developed in order to determine the position of the Arago neutral point, which could be used as an indicator of the turbidity level of the atmosphere by fully automating the functioning to reduce the time spent on taking a set of data, using colour filters to select narrow spectral bands, taking data from a higher altitude free of nearby buildings and trees, and using a detector with a linear response.

\section{Acknowledgement}

The authors would like to express their appreciation to Mr. O.K.D. Manoj Priyantha for his technical assistance in the mechanical construction of the polarimeter and to Mr. B.G.R. Amaranayake for his initial painstaking study of skylight polarisation using a manually operated polarimeter.

\section{REFERENCES}

1. Chandrasekhar S. \& Elbert D.D. (1954). The illumination and polarization of the sunlit sky on rayleigh scattering. Transactions of the American Philosophical Society. New Series 44(6): 643 - 654 .

2. Coulson K.L. (1980). Characteristics of skylight at the zenith during twilight as indicators of atmospheric turbidity. Applied Optics 19(20): 3469 - 8480. 
3. Gal J., Hovarth G., Benno Meyer-Rochow V. \& Wehner R. (2001). Polarization patterns of the summer sky and its neutral points measured by full-sky imaging polarimetry in Finnish Lapland north of the Arctic Circle. Proceedings of the Royal Society of London A 457: 1385 - 1399.

4. Gambling D.J. \& Billard B. (1967). A study of the polarization of skylight. Australian Journal of Physics 20: $675-681$.

DOI: http://dx.doi.org/10.1071/PH670675

5. Holzworth G.C. \& Rao C.R.N. (1965). Studies of skylight polarization. Journal of the Optical Society of America 55(4): $403-408$.

DOI: http://dx.doi.org/10.1364/JOSA.55.000403

6. Hovarth G., Bernath B., Suhai B. \& Barta A. (2002). First observation of the fourth neutral polarization point in the atmosphere. Journal of the Optical Society of America A 19(10): 2085 - 2099.

DOI: http://dx.doi.org/10.1364/JOSAA.19.002085
7. Hovarth G., Gal J. \& Pomozi I. (1998). Polarization portrait of the arago point: video-polarimetricimaging of the neutral points of skylight polarization. Naturwissenschaften 85: $333-339$.

DOI: http://dx.doi.org/10.1007/s001140050510

8. Konnen G.P. (1985). Polarized Light in Nature. Cambridge University Press, Cambridge, UK.

9. Liu Y. \& Voss K. (1997). Polarized radiance distribution measurement of skylight II experiment and data. Applied Optics 36(33): 8753 - 8764 .

DOI: http://dx.doi.org/10.1364/AO.36.008753

10. Sekera Z. (1957). Light scattering in the atmosphere and the polarization of skylight. Journal of the Optical Society of America 47(6): 484 - 490.

DOI: http://dx.doi.org/10.1364/JOSA.47.000484

11. Smith G.S. (2007). The polarization of skylight: an example from nature. American Journal of Physics 75(1): 25 - 35. DOI: http://dx.doi.org/10.1119/1.2360991 